\title{
Distributed Control and Energy Storage Requirements of Networked Dc Microgrids
}

\author{
Wayne W. Weaver ${ }^{\mathrm{a}}$, Rush D. Robinett, III ${ }^{\mathrm{a}}$, Gordon G. Parker ${ }^{\mathrm{a}}$, David G. \\ Wilson ${ }^{\mathrm{b}}$ \\ ${ }^{a}$ Michigan Technological University, 1400 Townsend Dr., Houghton, Michigan, 49931, \\ USA \\ ${ }^{b}$ Sandia National Laboratories, P.O. Box 5800, Albuquerque, New Mexico, 87185, USA
}

\begin{abstract}
Microgrids are a key technology to help improve the reliability of electric power systems and increase the integration of renewable energy sources. Interconnection and networking of smaller microgrids into larger systems have potential for even further improvements. This paper presents a novel approach to a distributed droop control and energy storage in networked dc microgrids. Distributed control is necessary to prevent single points of failure along with flexibility and adaptability to changing energy resources. The results show that systems with random sources and fast update rates, a networked microgrid structure can minimize required energy storage requirements.
\end{abstract}

Keywords: microgrid, distributed control, energy storage, optimization, power electronics

Email addresses: wwweaver@mtu.edu (Wayne W. Weaver), rdrobine@mtu.edu (Rush D. Robinett, III), ggparker@mtu .edu (Gordon G. Parker), dwilso@sandia.gov (David G. Wilson) 


\section{Introduction}

A microgrid is a collection of energy resources on a common network. These resources include generation, conversion, loads and storage devices [1]. The model of centralized generation is gradually being replaced by a distributed generation model [2]. The emerging technologies in renewable and distributed generation can have lower emissions and cost. The microgrid concept gives a solution for integration of a large number of distributed generations without causing disruption in the utility network. Microgrids also allow for local control of the distributed generation units and attests to the flexibility to operate autonomously during disturbances in the utility network to increase reliability $[3,4,5]$. In addition, the interconnection and networking of groups of microgrids can reduce the energy storage requirements. However, the interconnections and power flow control between microgrids increase costs, complexity and failure modes [6].

One of the main challenges for microgrid design and control is that generation capacity is very close to load demand. In addition, with the stochastic nature of most renewable energy sources there is a need for energy storage $[7,8,9]$. Energy storage can mitigate both long-term and short-term system transients. For example, a long term transient would be the generation variations over hours and days from a wind turbine or photo-voltaic array due to weather patterns. Short-term transients could include step changes in load or faults in the system where the response is on the order of seconds or fractions of a second. Therefore, a proper energy storage strategy will include devices that can respond at the proper bandwidth of system transients.

Within micorgrids, there are many approaches to the control and opti- 
mization of each element. A centralized approach is able to reach higher levels of performance at the cost of single points of failure and lack of flexibility. A distributed and de-centralized approach allows a very flexible system that can adapt to changing system structures and situations. A typical approach to distributed control is droop control $[10,11]$. In de microgrids, droop control is equivalent to creating a virtual impedance between the source and the bus such that the total load current is distributed to the sources based on the weighted sum of droop settings. The standard way to implement droop control is through duty cycle control of the dc/dc converter interface to the bus.

Since many renewable sources are dc, such as photovoltaics, they require additional power conversion to connect to an ac system. In addition, most electronic loads require a dc power conversion step and many energy storage technologies, such as batteries or super-capacitors are also dc. Therefore, a dc system is a viable option for power distribution in microgrids [12, 13]. However, a dc microgrid with a high penetration of renewable sources can require large energy storage capacity to maintain the system and to mitigate variations in the sources [14].

This paper presents an alternative approach that uses the local energy storage device at the source to actuate the droop control in local and networked microgrids. The duty cycles of the converters are updated on a periodic interval to only match the source voltage to the bus. This approach allows the requirements for energy storage in capacity and bandwidth to be studied and designed with variations in the renewable energy sources and load. The novelty of this approach is that the system is not actuated through 
the typical approach of feed-back control of duty cycle of the dc-dc converter, but through feed-back control of the storage devices in the system. Further, the feed-back process of the energy storage actuation is implemented through a distributed droop control. The duty cycles of the dc-dc converter are only updated on periodic intervals through a feed-forward process.

The paper will first present the electrical system model of the dc-dc boost coverer, energy storage devices and microgrid structure. Next, the controls are developed for the feed-forward control of the duty cycles and the feedback control of the energy storage devices. Then, the distributed droop control is shown. Finally the feed-forwad, feed-back and distributed droop are demonstrated through simulation of several operational senarios.

\section{Dc-dc converter and microgrid model with energy storage}

In de microgrids the interface to the distribution bus is through de to dc converters. If the bus voltage is higher than the sources, the interface converter will be in the form of a boost converter. In this paper, the sources will be paired with an energy storage device and therefore the boost converter will be bi-directional.

\subsection{Dc-dc Converter Model}

A bidirectional dc-dc converter circuit is shown in Fig. 1. The converter is implemented with a power pole of two power MOSFETs, which enable forward blocking and reverse current [15]. In this configuration the top switch state is defined as $q$ while the bottom switch is $1-q$, then the dynamic 
equations for the converter are

$$
\begin{aligned}
L \frac{d i_{L}}{d t} & =v_{s}-i_{L} R_{L}-q(t) v_{C} \\
C_{B} \frac{d v_{C}}{d t} & =q(t) i_{L}-i_{\text {Load }} .
\end{aligned}
$$

Formally, when $i_{L}>0$ this converter is a boost converter and when $i_{L}<0$ it is a buck converter. However, in this paper we will refer to the circuit as a boost, even though it can have bidirectional current. The time average of the switch state is found from

$$
\lambda=\frac{1}{T_{s w}} \int_{t-T_{s w}}^{t} q(t) d t
$$

where $T_{s w}$ is the switching period. The dynamic average model of the converter [16] is

$$
\begin{aligned}
L \frac{d i_{L}}{d t} & =v_{s}-i_{L} R_{L}-\lambda v_{C} \\
C_{B} \frac{d v_{C}}{d t} & =\lambda i_{L}-i_{\text {Load }} .
\end{aligned}
$$

This model of the converter ensures two quadrant operation because of the choice of the switches in Fig. 1 as, $v_{C}>v_{s}$ and bidirectional current $i_{L}$.

\subsection{Source and storage model}

Consider the bus interface boost converter shown in Fig. 2 which has two voltage sources. The voltage source $v_{v}$ represents an energy source such as a generator or photo-voltaic panel. The source $v_{u}$ represents an energy storage device such as a battery or capacitor. Both voltage sources include a 


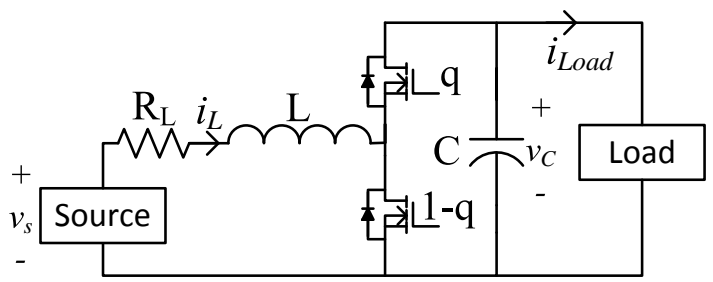

Figure 1: A bi-directional dc-dc converter.

series equivalent resistance $R_{v}$. Both the source and storage model in Fig. 2 represent a Thevenin equivalent of a source and a second stage converter such that the output terminal voltages are controllable. Both the source and storage converters will contribute current to the inductor of the bus interface boost converter. The total inductor current is

$$
i_{L}=\frac{v_{u}-v_{L}}{R_{u}}+\frac{v_{v}-v_{L}}{R_{v}}
$$

and the node voltage $v_{L}$ is

$$
v_{L}=v_{v} \frac{R_{u}}{R_{u}+R_{v}}+v_{u} \frac{R_{v}}{R_{u}+R_{v}}-i_{L} \frac{R_{u} R_{v}}{R_{u}+R_{v}}
$$

It is seen in (7) that the total voltage is a sum of two series sources and a line impedance. The voltages and resistances (7) can be lumped into the new 


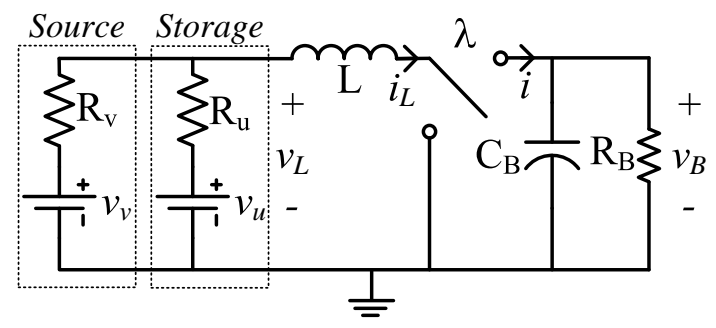

Figure 2: A boost converter model with two parrallel voltage sources, where $v_{v}$ represents an energy source and $v_{u}$ represents an energy storage device.

variables

$$
\begin{aligned}
v & =v_{v} \frac{R_{u}}{R_{u}+R_{v}} \\
u & =v_{u} \frac{R_{v}}{R_{u}+R_{v}} \\
R_{L} & =\frac{R_{u} R_{v}}{R_{u}+R_{v}} .
\end{aligned}
$$

The boost converter with energy source and storage devices can be modeled as a series combination in the new variables of voltage sources and Thevenin resistance as shown in boost converter models of Fig. 3 with the average mode [16] dynamic model

$$
\begin{aligned}
L \frac{d i_{L}}{d t} & =-\lambda v_{B}-i_{L} R_{L}+u+v \\
C_{B} \frac{d v_{B}}{d t} & =\lambda i_{L}-v_{B} \frac{1}{R_{B}}
\end{aligned}
$$

where $\lambda$ represents duty cycle of the converter.

\subsection{Microgrid model}

A simple dc microgrid model is shown in Fig. 3 where $N$ boost converters have the series model derived in section 2.2 for the source and local energy 


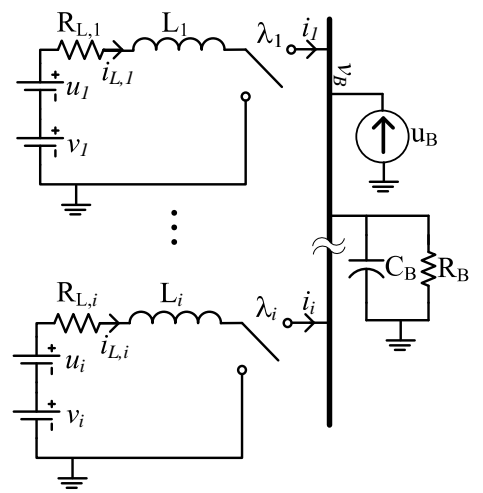

Figure 3: Microgrid of equivalent series boost converters.

storage devices. The model of the microgrid in Fig. 3 has the dynamic equations

$$
\begin{aligned}
L_{i} \frac{d i_{L, i}}{d t} & =-\lambda_{i} v_{B}-i_{L, i} R_{L, i}+u_{i}+v_{i}, \quad i=1 . . N \\
C_{B} \frac{d v_{B}}{d t} & =\sum_{i=1}^{N} \lambda_{i} i_{L, i}-v_{B} \frac{1}{R_{B}}+u_{B}
\end{aligned}
$$

where $u_{B}$ represents a centralized bus energy storage device. In implementation, $u_{B}$ would be an energy storage device, such as a battery or ultracapacitor, interfaced to the bus through a dc to de power converter. However, for this study it is important to represent the energy storage devices as ideal sources that are controlled to respond to system dynamics. In this way the storage requirements, such as total energy delivered or absorbed and response bandwidth can be determined. The microgrid load is modeled as a simple bus resistor and capacitor $R_{B}$ and $C_{B}$, so each microgrid will have $N+1$ states. The next challenge is to control the boost converters in such way as the load current is shared between the sources. In addition, because 
of highly variable sources $v_{i}$, which is indicative of renewable energy sources, storage at the local converters and central bus is required to maintain a nominal operating point of the bus voltage. In general, the optimal distribution, capacity and bandwidth of the energy storage devices is not well understood, especially for a distributed control architecture.

\subsection{Networked microgrid model}

Each microgrid as depicted in Fig. 3 is a self contained power system. However, due to the lack of diversity and inertia in the microgrid, its stability, reliability and flexibility may not be optimal. If multiple microgrids are interconnected then it may be possible to improve the reliability, flexibility and stability of the overall system. However energy storage remains a crucial element in the system. In addition, as the network of microgrids becomes larger, a distributed control architecture becomes even more attractive given that there is no need for a communication infrastructure or central controller. However, it is important to understand the stability margins and energy storage requirements in such a system.

Given a known set of sources and loads, there are numerous permutations of networked microgrids. In general, a networked microgrid will have a bus bar voltage vector $\mathbf{V}$, and an injected current vector $\mathbf{I}$ and an interconnection admittance matrix $\mathbf{Y}$. Then, the following matrix relationship can be written

$$
\mathbf{I}=\mathbf{Y V}
$$

where all injected current and voltage vectors are $k \times 1$, with $k$ representing the number of bus bars. The bus admittance matrix $\mathbf{Y}$ is symmetric in most 
cases and is a function of line admittances and shunt load resistances of the microgrid. The solution of the nodal equations in (15) follows the methodology discussed in [17]. In this paper, a simple common bus interconnected microgrid structure will be used.

Consider the networked microgrid configuration shown in Fig. 4 where there are $N$ sub-microgrids connected to a common bus. Each microgrid has a bus storage element $u_{B, k}$, an $R C$ load, and $M$ source converters. Power flow for interconnection of the buses is achieved through an interconnection converter from bus $k$ to network $n$ denoted $k \rightarrow n$ with local energy storage $u_{k \rightarrow n e t}$. In the configuration shown in Fig. 4 , the network bus net must be at a higher voltage than any of the lower microgrids $1 . . N$. In this configuration, power can directly flow from one microgrid to another. The networked microgrid Fig. 4 will have the system of equations

$$
\begin{gathered}
L_{k, m} \frac{d i_{L, k, m}}{d t}=-\lambda_{k, m} v_{B, k}-i_{L, k, m} R_{L, k, m}+u_{k, m}+v_{k, m}, \\
k=1 . . N, \quad m=1 . . M \\
C_{B, k} \frac{d v_{B, k}}{d t}=\sum_{m=1}^{M} \lambda_{k, m} i_{L, k, m}-i_{L, k \rightarrow n e t}-\frac{v_{B, k}}{R_{B, k}}+u_{B, k}, \\
k=1 . . N
\end{gathered}
$$




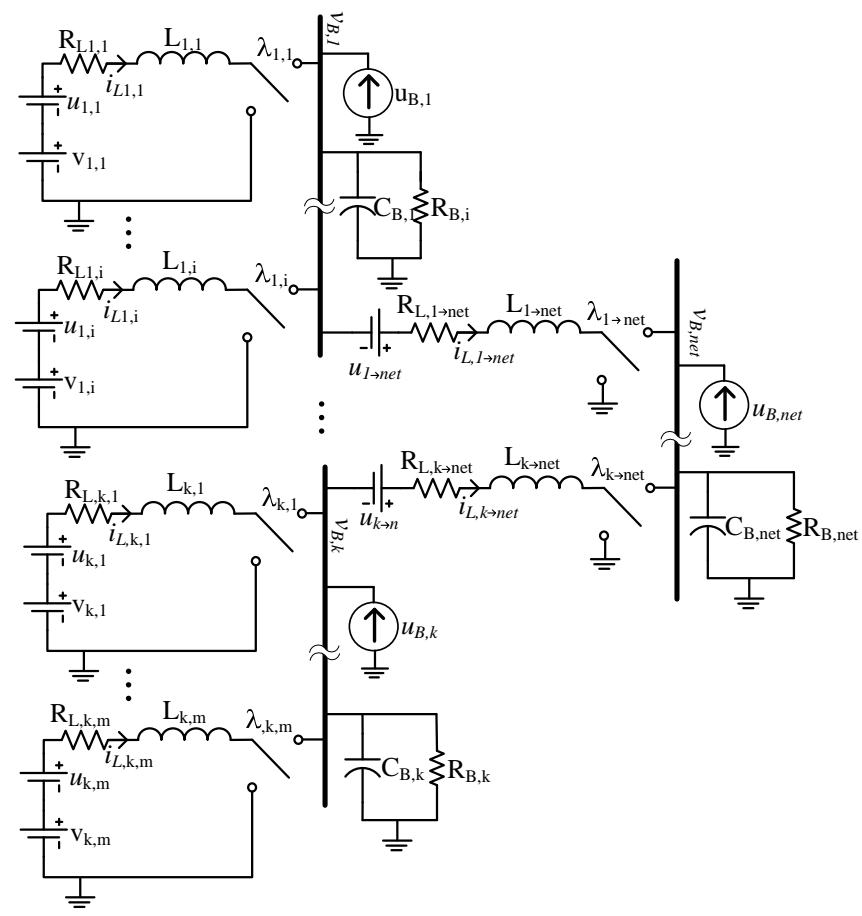

Figure 4: Network of $k$ microgrids connected to a common bus. Microgrid 1 has $i$ source converters and microgrid $k$ has $m$ source converters .

$$
\begin{gathered}
L_{k \rightarrow n e t} \frac{d i_{L, k \rightarrow n e t}}{d t}=-\lambda_{k \rightarrow n e t} v_{B, n e t}-i_{L, k \rightarrow n e t} R_{L, k \rightarrow n e t} \\
+u_{k \rightarrow n e t}+v_{B, k}, \\
k=1 . . N \\
C_{B, n e t} \frac{d v_{B, n e t}}{d t}=\sum_{i=1}^{N} \lambda_{i \rightarrow n e t} i_{L, i \rightarrow n e t} \\
-\frac{v_{B, n e t}}{R_{B, n e t}}+u_{B, n e t} .
\end{gathered}
$$




\section{Feed-forward duty cycle and feed-back energy stortage control}

For the control development consider the microgrid structure in Fig. 4 with 2 sub-microgrids $(N=2)$ and both microgrids have 2 boost converter sources $(M=2)$. Then the dynamic system equations (16) - (19) will have $N(2+M)+1=9$ states. In this paper, the duty cycles, $\lambda$, will only be updated on the interval $\Delta t_{\lambda}$, and held constant otherwise. This zero-order hold approach will mimic the effect of a discrete digital control system that may have limited computational power. Then, by using the zero-order hold approach, the effects of a digital control and information flow can be studied on the performance of the system. In this paper, it is desired to study a distributed control that will only require local information at the converter to determine the control action. However, a centralized approach based on system wide information can also be used $[3,9,6]$. The updated duty cycle commands are obtained from the steady-state solution of (16) and (18), with $u=0$, such that

$$
\begin{aligned}
\lambda_{k, m}= & \frac{1}{v_{B, k}}\left(v_{k, m}+i_{L, k, m} R_{L, k, m}\right), \\
& k=1 . . N, \quad m=1 . . M \\
\lambda_{k \rightarrow n e t}= & \frac{1}{v_{B, n e t}}\left(v_{B, k}+i_{L, k \rightarrow n e t} R_{L, k \rightarrow n e t}\right), \\
& k=1 . . N .
\end{aligned}
$$

The duty cycle update strategy for the source converters in (20) and the interface converter in (21) match the high side terminal voltage of the converter, the bus voltage, to the low side source voltage minus the energy storage 
voltage. This forces the energy storage device back to a zero output condition. The feed-forward control in (20) and (21) were chosen to remove the current load from the converters energy storage device. Between updates of the feed-forward duty cycle command the energy storage device actuates and enforces the reference command values. Since the boost converter duty cycle held constant between feed-forward updates, and not through feed-back, the problem of non-minimum phase in boost converters is eliminated [18]. To model the system in more general terms, a change of notation for the states is

$$
\begin{array}{ll}
\left(x_{11}, x_{12}, x_{21}, x_{22}\right) & =\left(i_{L, 1,1}, i_{L, 1,2}, i_{L, 2,1}, i_{L, 2,2}\right) \\
\left(x_{13}, x_{23}\right) & =\left(i_{L, 1 \rightarrow n e t}, i_{L, 2 \rightarrow n e t}\right) \\
\left(x_{1}, x_{2}, x_{3}\right) & =\left(v_{B, 1}, v_{B, 2}, v_{B, n e t}\right) .
\end{array}
$$

Then the state equations can be written in the compact form

$$
\mathbf{M} \dot{\mathbf{x}}=\mathbf{R x}+\mathbf{u}+\mathbf{v}=[\overline{\mathbf{R}}+\tilde{\mathbf{R}}] \mathbf{x}+\mathbf{u}+\mathbf{v}
$$

where

$$
\begin{aligned}
\mathbf{x} & =\left[x_{11}, x_{12}, x_{21}, x_{22}, x_{13}, x_{23}, x_{1}, x_{2}, x_{3}\right]^{T} \\
\mathbf{v} & =\left[v_{11}, v_{12}, v_{21}, v_{22}, 0,0,0,0,0\right]^{T} \\
\mathbf{u} & =\left[u_{11}, u_{12}, u_{21}, u_{22}, u_{1 \rightarrow 3}, u_{2 \rightarrow 3}, u_{B 1}, u_{B 2}, u_{B 3}\right]^{T} .
\end{aligned}
$$

The matrices from (23) for the example in Fig. 4 are 
$\overline{\mathbf{R}}=\left[\begin{array}{ccccccccc}-R_{L, 11} & 0 & 0 & 0 & 0 & 0 & 0 & 0 & 0 \\ 0 & -R_{L, 12} & 0 & 0 & 0 & 0 & 0 & 0 & 0 \\ 0 & 0 & -R_{L, 21} & 0 & 0 & 0 & 0 & 0 & 0 \\ 0 & 0 & 0 & -R_{L, 22} & 0 & 0 & 0 & 0 & 0 \\ 0 & 0 & 0 & 0 & -R_{L, 13} & 0 & 0 & 0 & 0 \\ 0 & 0 & 0 & 0 & 0 & -R_{L, 23} & 0 & 0 & 0 \\ 0 & 0 & 0 & 0 & 0 & 0 & -\frac{1}{R_{B 1}} & 0 & 0 \\ 0 & 0 & 0 & 0 & 0 & 0 & 0 & -\frac{1}{R_{B 2}} & 0 \\ 0 & 0 & 0 & 0 & 0 & 0 & 0 & 0 & -\frac{1}{R_{B 3}}\end{array}\right]$

$$
\tilde{\mathbf{R}}=\left[\begin{array}{ccccccccc}
0 & 0 & 0 & 0 & 0 & 0 & -\lambda_{11} & 0 & 0 \\
0 & 0 & 0 & 0 & 0 & 0 & -\lambda_{12} & 0 & 0 \\
0 & 0 & 0 & 0 & 0 & 0 & 0 & -\lambda_{21} & 0 \\
0 & 0 & 0 & 0 & 0 & 0 & 0 & -\lambda_{22} & 0 \\
0 & 0 & 0 & 0 & 0 & 0 & 0 & 0 & -\lambda_{13} \\
0 & 0 & 0 & 0 & 0 & 0 & 0 & 0 & -\lambda_{23} \\
\lambda_{11} & \lambda_{12} & 0 & 0 & 0 & 0 & 0 & 0 & 0 \\
0 & 0 & \lambda_{21} & \lambda_{22} & 0 & 0 & 0 & 0 & 0 \\
0 & 0 & 0 & 0 & \lambda_{13} & \lambda_{23} & 0 & 0 & 0
\end{array}\right]
$$

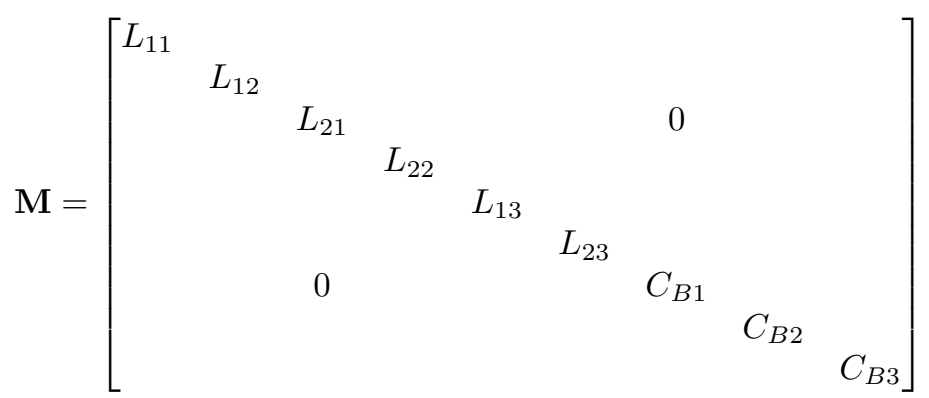

and the resistive matrices are shown in (28) and (29). 


\subsection{Hamiltonian surface shaping power flow control}

A key element of the proposed distributed droop control is a PI control strategy based on a Hamiltonian Surface Shaping Power Flow Control (HSSPFC) approach [19]. The first step is to define an error state for (23)

$$
\tilde{\mathbf{x}}=\mathbf{x}_{\mathrm{ref}}-\mathbf{x}
$$

where the reference state and control vectors are defined by

$$
\mathbf{M} \dot{\mathbf{x}}_{\mathrm{ref}}=[\overline{\mathbf{R}}+\tilde{\mathbf{R}}] \mathbf{x}_{\mathrm{ref}}+\mathbf{u}_{\mathrm{ref}}+\mathbf{v} .
$$

The reference vector $\mathbf{x}_{\mathbf{r e f}}$ is the set of nominal state variables. Some of the nominal values, such as the nominal bus voltage is set by the system designer. The other reference states, such as the converter currents, are set through the droop control calculations as will be discussed in section 4 . The next step is to define the Hamiltonian as

$$
\mathbf{H}=\frac{1}{2} \tilde{\mathbf{x}}^{T} \mathbf{M} \tilde{\mathbf{x}}+\frac{1}{2}\left(\int_{0}^{t} \tilde{\mathbf{x}}^{T} d \tau\right) \mathbf{K}_{\mathbf{I}}\left(\int_{0}^{t} \tilde{\mathbf{x}} d \tau\right)
$$


which is positive definite about $\int \tilde{\mathbf{x}}=\mathbf{0}$ for $\mathbf{M}$ and $\mathbf{K}$ positive definite and is the static stability condition. The time derivative of (32) is

$$
\begin{aligned}
\dot{\mathbf{H}}= & \tilde{\mathbf{x}}^{T} \mathbf{M} \tilde{\dot{\mathbf{x}}}+\tilde{\mathbf{x}}^{T} \mathbf{K}_{I} \int_{0}^{t} \tilde{\mathbf{x}} d \tau \\
= & \tilde{\mathbf{x}}^{T}\left[\mathbf{M} \dot{\mathbf{x}}_{\text {ref }}-\mathbf{M} \dot{\mathbf{x}}\right]+\tilde{\mathbf{x}}^{T} \mathbf{K}_{I} \int_{0}^{t} \tilde{\mathbf{x}} d \tau \\
= & \tilde{\mathbf{x}}^{T}\left[(\overline{\mathbf{R}}+\tilde{\mathbf{R}}) \mathbf{x}_{\text {ref }}+\mathbf{u}_{\mathbf{r e f}}+\mathbf{v}-(\overline{\mathbf{R}}+\tilde{\mathbf{R}}) \mathbf{x}-\mathbf{u}-\mathbf{v}\right] \\
& +\tilde{\mathbf{x}}^{T} \mathbf{K}_{I} \int_{0}^{t} \tilde{\mathbf{x}} d \tau \\
= & \tilde{\mathbf{x}}^{T}(\overline{\mathbf{R}}+\tilde{\mathbf{R}}) \tilde{\mathbf{x}}+\tilde{\mathbf{x}}^{T}\left(\mathbf{u}_{\mathbf{r e f}}-\mathbf{u}\right)+\tilde{\mathbf{x}}^{T} \mathbf{K}_{I} \int_{0}^{t} \tilde{\mathbf{x}} d \tau \\
= & \tilde{\mathbf{x}}^{T} \overline{\mathbf{R}} \tilde{\mathbf{x}}+\tilde{\mathbf{x}}^{T} \mathbf{\Delta} \mathbf{u}+\tilde{\mathbf{x}}^{T} \mathbf{K}_{I} \int_{0}^{t} \tilde{\mathbf{x}} d \tau
\end{aligned}
$$

since $\tilde{\mathbf{x}}^{T} \tilde{\mathbf{R}} \tilde{\mathbf{x}}=0$.

Now, select a proportional-integral (PI) controller as

$$
\Delta \mathbf{u}=-\mathbf{K}_{\mathbf{P}} \tilde{\mathbf{x}}-\mathbf{K}_{\mathbf{I}} \int_{0}^{t} \tilde{\mathbf{x}} d \tau
$$

which gives

$$
\mathbf{u}=\mathbf{u}_{\mathrm{ref}}-\Delta \mathbf{u}
$$

and

$$
\dot{\mathbf{H}}=-\tilde{\mathbf{x}}^{T}\left[\mathbf{K}_{\mathbf{P}}-\overline{\mathbf{R}}\right] \tilde{\mathbf{x}}<0
$$

where $\mathbf{K}_{\mathbf{P}}$ and $\mathbf{K}_{\mathbf{I}}$ are positive definite controller gain matrices. Equation (36) enables a guideline for picking the gains of the PI controller to maintain 
stability and performance. For (36), only $\dot{\mathbf{H}}(\tilde{\mathbf{x}}=0)=0$. However, this only proves stability for the state variables $\tilde{\mathbf{x}}$. Since the control dynamics of $\int_{0}^{t} \tilde{\mathbf{x}} d \tau$ are not included, further analysis is needed to prove asymptotic stability.

\subsection{Feed-back control dynamics stability}

The stability of the feed-back PI control dynamics can be found from the higher order derivatives of the Hamiltonian in (32) [20].

Theorem Assume there exists a Lyapunov function $V(x)$ of the dynamical system $\dot{\mathbf{x}}=f(\mathbf{x})$. Let $\Omega$ be a non-empty set of the state vectors such that

$$
\mathbf{x} \in \Omega \Rightarrow \dot{V}(\mathbf{x})=0
$$

If the first $k-1$ derivatives of $V(\mathbf{x})$, evaluated on the set $\Omega$, are zero

$$
\frac{d^{i} v(\mathbf{x})}{d \mathbf{x}^{i}}=0 \quad \forall \mathbf{x} \in \Omega \quad i=1,2, \ldots, k-1
$$

and the $k$-th derivative is negative definite on the set $\Omega$

$$
\frac{d V(\mathbf{x})}{d \mathbf{x}}<0 \quad \forall \mathbf{x} \in \Omega
$$

then the system $\mathbf{x}(t)$ is asymptotically stable, if $k$ is an odd number.

The feed-forward and feed-back PI control law is

$$
\mathbf{u}=\mathbf{u}_{\text {ref }}-\Delta \mathbf{u}=\mathbf{M} \dot{\mathbf{x}}_{\text {ref }}-\mathbf{R} \mathbf{x}_{\text {ref }}-\mathbf{v}+\mathbf{K}_{\mathbf{P}} \tilde{\mathbf{x}}+\mathbf{K}_{\mathbf{I}} \int_{0}^{t} \tilde{\mathbf{x}} d \tau
$$


where $u_{r e f}$ is found from the solution of (31). Then the system state trajectories are

$$
\begin{aligned}
\mathbf{M} \dot{\mathbf{x}} & =\mathbf{R} \mathbf{x}+\mathbf{v}+\mathbf{u} \\
& =\mathbf{R x}+\mathbf{v}+\mathbf{M} \dot{\mathbf{x}}_{\mathbf{r e f}}-\mathbf{R x}_{\mathrm{ref}}-\mathbf{v}+\mathbf{K}_{\mathbf{P}} \tilde{\mathbf{x}}+\mathbf{K}_{\mathbf{I}} \int_{0}^{t} \tilde{\mathbf{x}} d \tau
\end{aligned}
$$

The deviation in the state trajectories are

$$
\mathbf{M} \dot{\tilde{\mathbf{x}}}=\left[\mathbf{R}-\mathbf{K}_{\mathbf{P}}\right] \tilde{\mathbf{x}}-\mathbf{K}_{\mathbf{I}} \int_{0}^{t} \tilde{\mathbf{x}}^{T} d \tau
$$

then

$$
\dot{\tilde{\mathbf{x}}}=\mathbf{M}^{-1}\left[\left(\mathbf{R}-\mathbf{K}_{\mathbf{P}}\right) \tilde{\mathbf{x}}-\mathbf{K}_{\mathbf{I}} \int_{0}^{t} \tilde{\mathbf{x}} d \tau\right]
$$

The second time derivative of the Hamiltonian is

$$
\begin{aligned}
\ddot{\mathbf{H}} & =-2 \tilde{x}^{T}\left(\mathbf{K}_{\mathbf{P}}-\overline{\mathbf{R}}\right) \dot{\tilde{x}} \\
& =-2 \tilde{\mathbf{x}}^{T}\left(\mathbf{K}_{\mathbf{P}}-\overline{\mathbf{R}}\right) \mathbf{M}^{-1}\left[\left(\mathbf{R}-\mathbf{K}_{\mathbf{P}}\right) \tilde{\mathbf{x}}-\mathbf{K}_{\mathbf{I}} \int_{0}^{t} \tilde{\mathbf{x}} d \tau\right] \\
& =0 \quad \text { for } \quad \tilde{\mathbf{x}}=0 .
\end{aligned}
$$


The third oder time derivative of the Hamiltonian from (32) is

$$
\begin{aligned}
\dddot{\mathbf{H}}= & -2 \dot{\tilde{\mathbf{x}}}^{T}\left(\mathbf{K}_{\mathbf{P}}-\overline{\mathbf{R}}\right) \mathbf{M}^{-1}\left[\left(\mathbf{R}-\mathbf{K}_{\mathbf{P}}\right) \tilde{\mathbf{x}}-\mathbf{K}_{\mathbf{I}} \int_{0}^{t} \tilde{\mathbf{x}} d \tau\right] \\
- & 2 \tilde{\mathbf{x}}^{T}\left(\mathbf{K}_{\mathbf{P}}-\overline{\mathbf{R}}\right) \mathbf{M}^{-1}\left[\left(\mathbf{R}-\mathbf{K}_{\mathbf{P}}\right) \dot{\tilde{\mathbf{x}}}-\mathbf{K}_{\mathbf{I}} \tilde{\mathbf{x}}\right] \\
=- & 2\left[\mathbf{M}^{-1}\left[\left(\mathbf{R}-\mathbf{K}_{\mathbf{P}}\right) \tilde{\mathbf{x}}-\mathbf{K}_{\mathbf{I}} \int_{0}^{t} \tilde{\mathbf{x}} d \tau\right]\right]^{T}\left(\mathbf{K}_{\mathbf{P}}-\overline{\mathbf{R}}\right) \mathbf{M}^{-1} \\
& {\left[\left(\mathbf{R}-\mathbf{K}_{\mathbf{P}}\right) \tilde{\mathbf{x}}-\mathbf{K}_{\mathbf{I}} \int_{0}^{t} \tilde{\mathbf{x}} d \tau\right] } \\
- & 2 \tilde{\mathbf{x}}^{T}\left[\mathbf{K}_{\mathbf{P}}-\overline{\mathbf{R}}\right] \mathbf{M}^{-1} \\
& {\left[\left(\mathbf{R}-\mathbf{K}_{\mathbf{P}}\right) \mathbf{M} \mathbf{M}^{-1}\left[\left(\mathbf{R}-\mathbf{K}_{\mathbf{P}}\right) \tilde{\mathbf{x}}-\mathbf{K}_{\mathbf{I}} \int_{0}^{t} \tilde{\mathbf{x}} d \tau\right]-\mathbf{K}_{\mathbf{I}} \tilde{\mathbf{x}}\right] } \\
=- & {\left[\mathbf{M}^{-1} \mathbf{K}_{\mathbf{I}} \int_{0}^{t} \tilde{\mathbf{x}} d \tau\right]^{T}\left[\mathbf{K}_{\mathbf{P}}-\overline{\mathbf{R}}\right]\left[\mathbf{M}^{-1} \mathbf{K}_{\mathbf{I}} \int_{0}^{t} \tilde{\mathbf{x}} d \tau\right]<0 }
\end{aligned}
$$

and

$$
\dddot{\mathbf{H}}=0 \quad \text { for } \quad \int_{0}^{t} \tilde{\mathbf{x}} d \tau=0 \text {. }
$$

Therefore the control dynamics are asymptotically stable. Furthermore proof of control dynamic stability can also be found in $[21,20]$.

\section{Droop control}

When two or more sources inject power into a common bus or grid a load sharing control scheme is required. If each source tries to control the bus voltage, or frequency in ac systems, then large circulation currents can result. A load sharing scheme can be centralized if an interconnected communication system is present and signals can be passed between sources to balance the 
load current. However, this can create a single point of failure. Droop control is a common technique for distributed control of electrical sources in a microgrid where the control implements a virtual impedance such that the load current is distributed between the sources proportional to the droop settings $V_{r e f, i}$ and $R_{d, i}$ [14]. The equivalent boost converter under droop control is shown in Fig. 5, where $V_{r e f, i}$ and $R_{d, i}$ are parameter settings for the control.

To implement the control in a source, and to control the bus voltages through the actuation of the energy storage devices, the reference for the bus current injection in the $i^{\text {th }}$ converter in Fig. 3 , is defined as

$$
i_{r e f, i}=\frac{V_{r e f, i}-v_{B}}{R_{d, i}} .
$$

An error signal is created from the reference inductor current as

$$
e_{i}=i_{r e f, i}-i_{i} .
$$

A control law to drive the error of (48) to zero is needed. However, it is important to point out that the control inputs to this model are the energy storage devices $u_{i}$ for the source converters and $u_{B, k}$ for the bus storage elements. This droop controller implements a decentralized version of the centralized feed-forward control of section 3.1 and utilizes the decoupled feedback control. The droop control will be actuated by the storage elements while the duty cycles $\lambda_{i}$ will be considered as a constant during a discrete epoch. The duty cycles will then be updated at set intervals as discussed in section 3. 
For the source converter the current injected into the bus is

$$
i_{i}=i_{L, i} \lambda_{i}
$$

with the error signal for the boost converters as

$$
e_{i}=i_{r e f, i}-i_{L, i} \lambda_{i}
$$

A proportional-integral control law for the droop control actuated by the energy storage device is

$$
u_{k, m}=R_{L, k, m} i_{r e f, k, m}+\lambda_{i} v_{B, k}-v_{k, m}+\left(K_{p, i} e_{i}+K_{i, i} \int e_{i} d t\right)
$$

where $u_{k, m} \in \mathbf{u}$ from (40). The droop control law for the bus storage devices is

$$
u_{B, j}=\frac{V_{r e f, i}-v_{B, k}}{R_{d, i}}
$$

The power from the boost storage devices

$$
p_{k, m}=u_{k, m} i_{L, k, m}
$$

The power from the bus storage devices

$$
p_{B, j}=u_{B, j} v_{B, k} .
$$




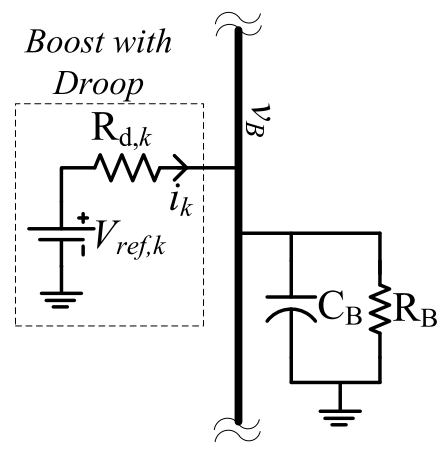

Figure 5: Equivalent terminal characteristics of a boost converter in a dc microgrid under voltage droop control.

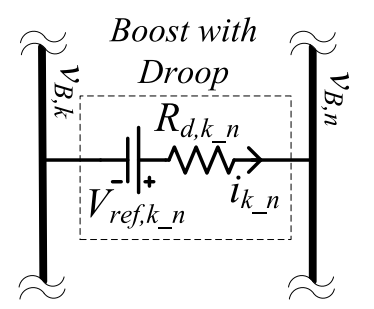

Figure 6: Equivalent terminal characteristics the interconnected bus boost converter.

The energy supplied from the storage device is

$$
w_{u}=\int p_{u} s t
$$

The boost converters that interconnect the buses in the microgrid also need a distributed control law to maintain bus voltages. Droop control can also be applied to these converter, however, the voltage must be references to the lower voltage bus. The terminal characteristics of the interconnecting converter are shown in Fig. 6 where $v_{B, n}>v_{B, k}$. 


\section{Simulation examples}

To demonstrate the distributed droop control approach to networked dc microgrids, a model was built and simulated in Wolfram Mathematica, Wolfram SystemModeler and Modelica [22]. The system shown in Fig. 4 has 2 sub-microgrids $(N=2)$ and both microgrids have 2 boost converter sources $(M=2)$. The sub-microgrids 1 and 2 have a nominal bus voltage of $100 \mathrm{~V}$ and the network bus 3 has a nominal voltage of $200 \mathrm{~V}$. All voltage sources $\left(v_{11}, v_{12}, v_{21}, v_{22}\right)$ have an average dc voltage of $48 \mathrm{~V}$. However, all these voltages also have uniform random white noise sampled on 1 second intervals superimposed on the dc voltage. This random noise input tests the performance of the proposed controller under non-ideal conditions that would be an extreme worst-case scenario for a field deployed microgrid. In microgrid applications the renewable sources are stochastic, but would be better behaved with lower bandwidth and magnitude variations in the transients than what is tested in this paper [23]. Therefore, the simulations shown in this section demonstrate the proposed control is a viable solution for field deployed microgrids.

The sources in microgrid 1 represent well behaved devices and are representative of dispachable sources such as diesel generators with very little voltage variation, in this example less than $2 \mathrm{~V}$. The sources in microgrid 2 represent highly variable sources and are representative of renewable sources such as photovoltaic and wind with large voltage variation, in this example up to $45 \mathrm{~V}$. The resistive loads on each sub-microgrid are $R_{B, 1}=R_{B, 2}=10 \Omega$ and the load on the network bus is $R_{B, 3}=100 \Omega$. The droop voltage settings $V_{\text {ref,i }}$ have been set to the respective bus nominal voltages. The boost con- 
verter droop resistances are set to $R_{d, i}=0.5 \Omega$, and the bus storage droop resistances are $2 \Omega$. The PI control gains were chosen to meet the requirements defined in section 3 and were picked to be $K_{P}=50, K_{I}=200$ for all controllers in the system, which were chosen to satisfy (36).

The structure and parameters of this example system were chosen to be indicative of many applications of networked microgrids. Microgrid applications such as military forward operating bases and electric ships have a zone based architecture [24]. Each zone of a microgrid can be a self contained power distribution microgrid of sources, loads and storage. Another way to view the zonal microgrid is as a network of smaller microgrids [25]. However, by interconnecting the zones or networked microgrids, greater reliability and potentially lower energy storage requirements can be achieved.

\subsection{Constant load example}

A short $10 \mathrm{~s}$ simulation example was run with constant loads and random voltage sources. The results from microgrid 2 are shown in Figs. 7-12. The random white noise voltage sources are shown in Fig. 7. The boost energy storage device voltages are shown in Fig. 8 with the duty cycles shown in Fig. 9. The boost energy storage device current and powers are shown in Fig. 10 and Fig. 11 respectively. The resulting bus 2 voltage is seen in Fig. 12. The rate at which all duty cycles are updated in this simulation example is $0.1 \mathrm{~s}$. As can be seen in Figs. 7-12, the bus voltages quickly return to their reference values set through the droop control when the source voltage variations occur.

It is seen in Fig. 12 that the bus voltage has an approximate average of $97.487 \mathrm{~V}$ which is less than the nominal $100 \mathrm{~V}$ due to droop control. It is also 


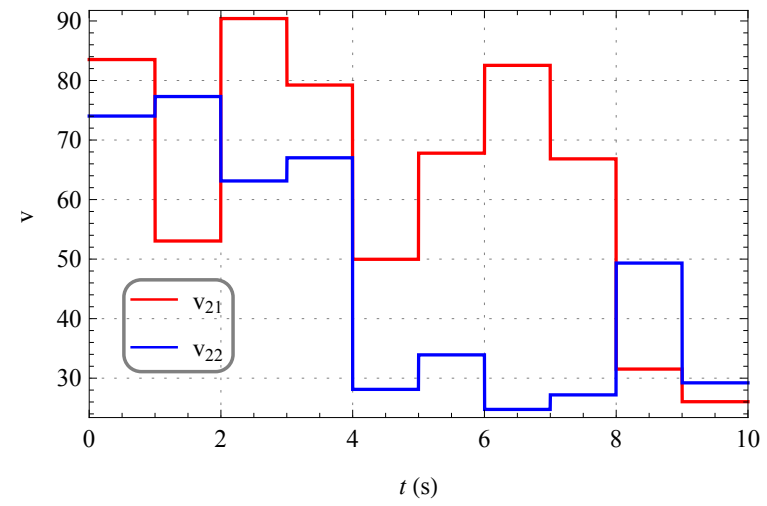

Figure 7: Boost converter source voltages.

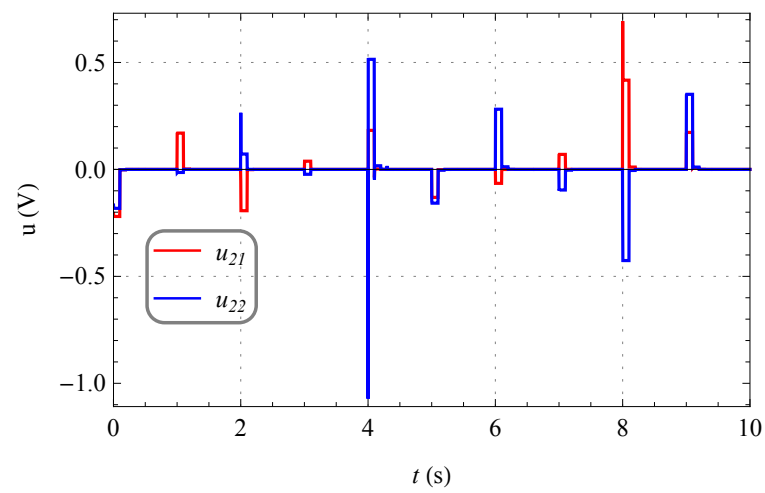

Figure 8: Boost converter storage device voltages.

seen that the bus voltage varies little when the source voltages vary because the boost converter energy storage devices act to maintain the desired droop characteristics. When the duty cycle is updated every $0.1 \mathrm{~s}$, the energy storage device voltage returns to zero along with the power output.

\subsection{Step change in load example}

To demonstrate the distributed droop control in networked microgrids, the same model from the previous section was modified such that the resistive 


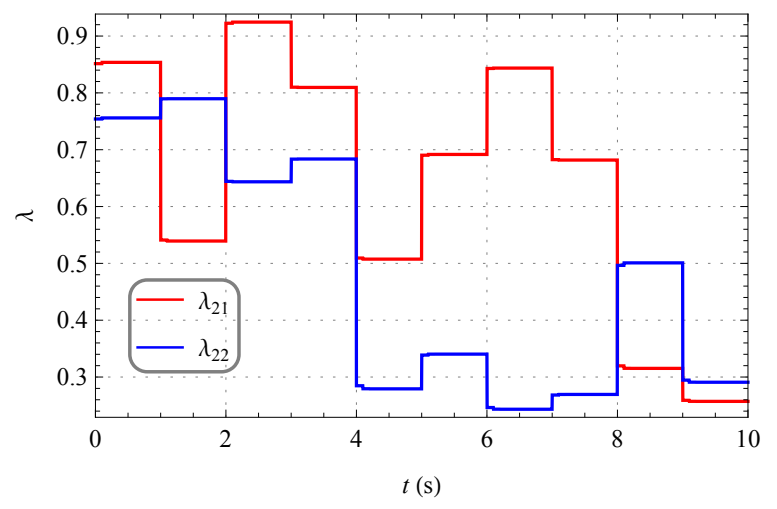

Figure 9: Boost converter duty cycles.

load on bus 2 is

$$
R_{B, 2}(t)=\left\{\begin{array}{ll}
10 \Omega, & \text { if } t<10 \mathrm{~s} \\
1 \Omega, & \text { if } t \geq 10 \mathrm{~s}
\end{array} .\right.
$$

The step change in load from (56) causes a transient in the system which draws more current from the sources. Through the droop control, the extra load current is shared between the boost converters and energy bus storage devices. The amount of current each device contributes is negotiated through the bus voltages by means of the droop control strategy. The following examples will show the performance of the system when each bus is isolated versus when they are interconnected.

\subsubsection{Isolated microgrids}

In the first simulation with the step change in load at bus 2, each bus is isolated from each other. This is accomplished through a very large droop setting of the interconnecting converters. In this case, no energy is shared between buses. The resulting sub-microgrid bus voltages are seen in Fig. 13, where the bus 2 voltage droops from $97.5 \mathrm{~V}$ down to $81.6 \mathrm{~V}$, while the bus 1 
voltage remains constant.

The energy from the local energy storage devices in microgrid 1 are shown in Fig. 14. The energy from the local energy storage devices in microgrid 2 are shown in Fig. 15. In addition, Fig. 16 shows that bus storage device 2 must output energy when the load steps. It is also seen in Fig. 16 that the bus storage device 3 is always providing energy to bus 3 , since in the isolated configuration, it is the only source of energy.

\subsubsection{Networked microgrids}

Next, the interconnecting converters were controlled to maintain droop resistances of $0.5 \Omega$, and thus allowing energy exchange between buses. It is seen in Fig. 17 and Fig. 18 that all three bus voltages droop in response to the increased load. It is important to point out that the change in the bus 2 voltage is now only approximately $85.6 \mathrm{~V}$, or $4 \mathrm{~V}$ less than in the previous isolated case.

The energy from the local boost energy storage devices in microgrid 1 and 2 are shown in Fig. 19 and Fig. 20 respectively. The energy from the bus storage devices is shown in Fig. 21. The energy from the interconnecting boost converters is shown in Fig. 22. It is seen in Fig. 22 that the storage devices in the interconnection converters contribute nor draw any significant amount energy, at least in comparison to the source and bus storage devices. This indicates that in the interconnection storage device may not be necessary in future iterations of a network microgrid design.

The performance and energy storage requirements of the system are greatly affected by the networked interconnection. Table 1 provides the a comparison of the total energy used as well as the maximum bus voltage 
Table 1: Total Energy and Voltage Droop Comparison

\begin{tabular}{lll}
\hline & Networked & Isolated \\
\hline$\sum W_{u}(\mathrm{~kJ})$ & 79.75 & 119.68 \\
\hline $\max \Delta V_{B}$ & 12.36 & 16.42 \\
\hline $\operatorname{avg} \Delta V_{B}$ & 6.35 & 5.56 \\
\hline
\end{tabular}

droop and average voltage droop from the networked and isolated simulation examples provided. Table 1 shows that in the networked configuration a total of only $79.75 \mathrm{~kJ}$ of energy was needed, while in the isolated case $119.68 \mathrm{~kJ}$ was needed. It is also seen in Table 1 that the average voltage droop changes very little between cases. These results lead to the conclusion if this system were to be built, smaller energy storage devices can be used, if the sub-microgrids can be networked together.

\subsubsection{Duty cycle feed-forward update rates}

Lastly, a series of simulations were performed to determine the effect of duty cycle update rate on the total energy required. In these simulations the models from section 5.2.1 and 5.2.2 was swept with duty cycle update rates of $\Delta t_{\lambda}=0.1 \mathrm{~s}$ to $1 \mathrm{~s}$ at steps of $0.001 \mathrm{~s}$. The total energy supplied by all the energy storage devices in the system for the networked and isolated cases are shown in Fig. 23. The results in Fig. 23 show that for small update rates, the energy required by the isolated case are greater than the networked case. As the time between updates increases, the isolated grid storage utilization approaches that of the networked grid. A possible explanation is that as the update period increases, the advantages of the interconnectivity degrade and the grid operates as a set of isolated microgrids. It's interesting to note that for both the interconnected and isolated cases the storage usage decreases as 
the duty cycle update increases. Based on the results of section 5.2.1 and 5.2.2 the bus voltage droop for the interconnected topology was lower than the grid's isolated operation. Extending that result to the update period study, the decrease in storage usage for longer update periods comes with a reduction in bus voltage performance.

\section{Conclusions}

This paper has presented a novel approach to droop control actuated by the local energy storage device. The novelty of this approach lies in the actuation of the system through the energy storage devices. The duty cycles are updated at periodic intervals through feed-forward control to match the high side and low side source voltages. This approach allows the storage needs for capacity and bandwidth response to be identified for the given distributed droop control approach. The results show that the networked microgrids need overall less energy storage in response to transients at certain duty cycle update rates. However, as the update rates decrease, the storage requirements lessen and become the same for a networked system versus isolated systems.

\section{Acknowledgment}

Sandia National Laboratories is a multiprogram laboratory operated by Sandia Corporation, a Lockheed Martin Company, for the U.S. Department of Energy's National Nuclear Security Administration under contract DEAC04-94AL85000. 


\section{References}

[1] R. H. Lasseter, Microgrids, in: IEEE Power Engineering Society Winter Meeting, Vol. 1, IEEE, 2002, pp. 305-308.

[2] K. A. Nigim, W.-J. Lee, Micro grid integration opportunities and challenges, in: IEEE Power Engineering Society General Meeting, IEEE, 2007, pp. 1-6.

[3] E. Trinklein, G. Parker, W. Weaver, R. Robinett, L. Babe, C.-W. Ten, W. Bower, S. Glover, S. Bukowski, Scoping study: Networked microgrids, Tech. Rep. Sandia Report SAND2014-17718, Sandia National Labs (2014).

[4] V. Ramirez, R. Ortega, O. Bethoux, A. Sánchez-Squella, A dynamic router for microgrid applications: Theory and experimental results, Control Engineering Practice 27 (0) (2014) 23 - 31.

[5] M. Erol-Kantarci, B. Kantarci, H. Mouftah, Reliable overlay topology design for the smart microgrid network, IEEE Network 25 (5) (2011) $38-43$.

[6] R. D. Robinett, D. G. Wilson, S. Y. Goldsmith, Collective control of networked microgrids with high penetration of variable resources part i: Theory, in: IEEE International Conference on Cyber Technology in Automation, Control, and Intelligent Systems, 2012, pp. 1-4.

[7] A. Chaouach, R. M. Kamel, R. Andoulsi, K. Nagasaka, Multiobjective intelligent energy management for a microgrid, IEEE Transactions on Industrial Electronics 60 (2013) 1688-1699. 
[8] M. H. Nazari, M. Ilic, J. Lopes, Small-signal stability and decentralized control design for electric energy systems with a large penetration of distributed generators, Control Engineering Practice 20 (9) (2012) 823831.

[9] D. G. Wilson, R. D. Robinett, S. Y. Goldsmith, Renewable energy microgrid control with energy storage integration, in: International Symposium on Power Electronics, Electrical Drives, Automation and Motion, 2012, pp. 158-163.

[10] B. K. Johnson, R. H. Lasseter, F. L. Alvarado, R. Adapa, Expandable multiterminal dc systems based on voltage droop, IEEE Transactions on Power Delivery 8 (4) (1993) 1926-1932.

[11] A. Nagliero, R. Mastromauro, D. Ricchiuto, M. Liserre, M. Nitti, Gainscheduling-based droop control for universal operation of small wind turbine systems, in: IEEE International Symposium on Industrial Electronics, IEEE, 2011, pp. 1459-1464.

[12] J. Guerrero, P. C. Loh, T.-L. Lee, M. Chandorkar, Advanced control architectures for intelligent microgrids - part ii: Power quality, energy storage, and ac/dc microgrids, IEEE Transactions on Industrial Electronics 60 (4) (2013) 1263-1270.

[13] A. M. Dizqah, A. Maheri, K. Busawon, P. Fritzson, Standalone de microgrids as complementarity dynamical systems: Modeling and applications, Control Engineering Practice 35 (0) (2015) 102 - 112. 
[14] W. W. Weaver, R. D. Robinett, G. G. Parker, D. G. Wilson, Energy storage requirements of dc microgrids with high penetration renewables under droop control, International Journal of Electrical Power \& Energy Systems 68 (0) (2015) $203-209$.

[15] N. Mohan, T. Undeland, Power Electronics: Converters, Applications, and Design, John Wiley \& Sons, 2007.

[16] P. T. Krein, J. Bentsman, R. M. Bass, B. L. Lesieutre, On the use of averaging for the analysis of power electronic systems, IEEE Transactions on Power Electronics 5 (2) (1990) 182-190.

[17] W. W. Weaver, P. T. Krein, Game-theoretic control of small-scale power systems, IEEE Transactions on Power Delivery 24 (3) (2009) 1560-1567.

[18] Z. Chen, W. Gao, J. Hu, X. Ye, Closed-loop analysis and cascade control of a nonminimum phase boost converter, IEEE Transactions on Power Electronics 26 (4) (2011) 1237-1252.

[19] R. D. Robinett III, D. G. Wilson, Nonlinear Power Flow Control Design: Utilizing Exergy, Entropy, Static and Dynamic Stability, and Lyapunov Analysis, Springer, 2011.

[20] H. Schaub, J. Junkins, Analytical mechanics of space systems, Aiaa, 2003.

[21] R. Robinett, G.G., Parker, H. Schaub, J. Junkins, Lyapunov optimal saturated control for nonlinear systems, Journal of Guidance, Control and Dynamics 20 (6) (1997) 1083-1088. 
[22] P. Fritzson, Introduction to modeling and simulation of technical and physical systems with Modelica, Wiley. com, 2011.

[23] A possibilistic probabilistic tool for evaluating the impact of stochastic renewable and controllable power generation on energy losses in distribution networks, a case study, Renewable and Sustainable Energy Reviews 15 (1) (2011) $794-800$.

[24] D. Wilson, J. Neely, M. Cook, S. Glover, J. Young, R. Robinett, Hamiltonian control design for dc microgrids with stochastic sources and loads with applications, in: IEEE International Symposium on Power Electronics, Electrical Drives, Automation and Motion, 2014, pp. 1264-1271.

[25] R. Robinett, D. Wilson, S. Goldsmith, Collective control of networked microgrids with high penetration of variable resources part i: Theory, in: IEEE International Conference on Cyber Technology in Automation, Control, and Intelligent Systems, 2012, pp. 1-4. 


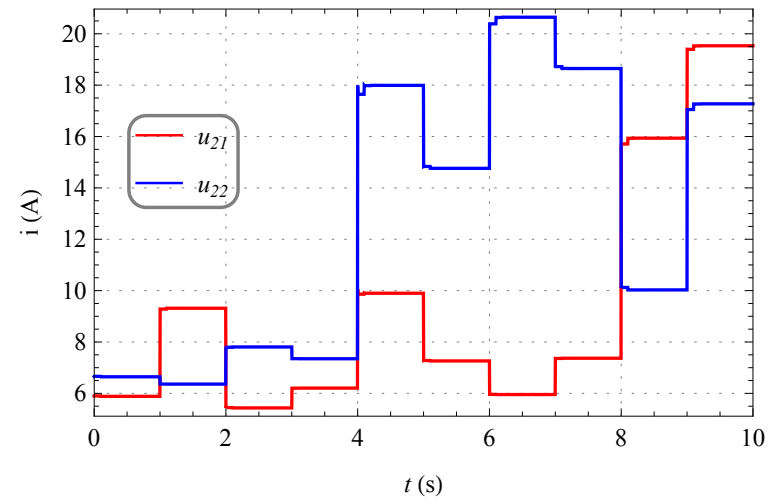

Figure 10: Boost converter storage device currents.

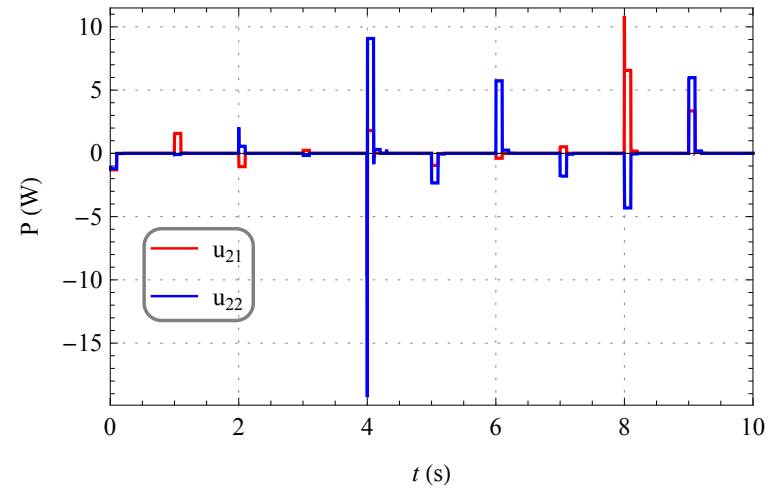

Figure 11: Boost converter storage device power.

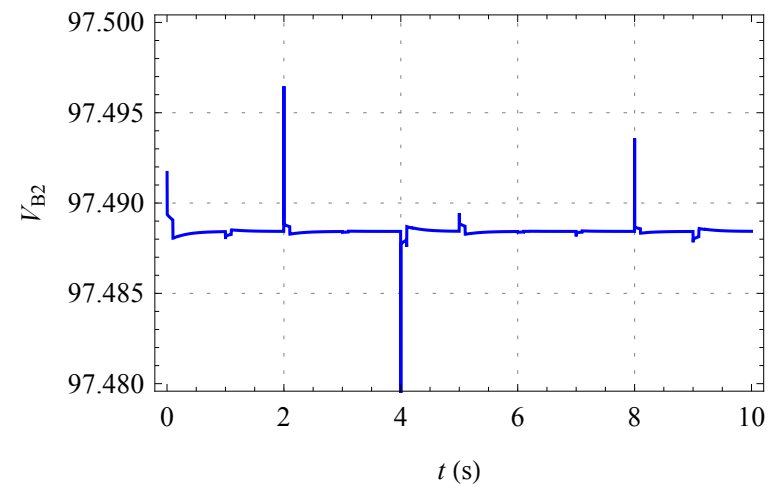

Figure 12: Bus 2 voltage. 


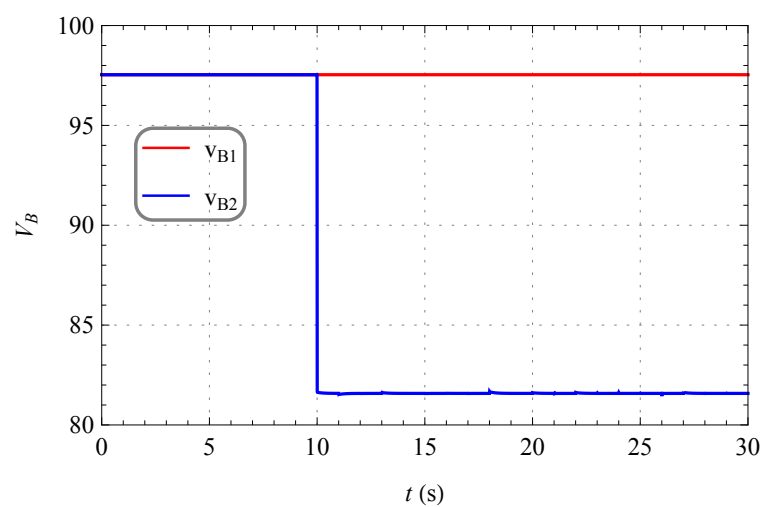

Figure 13: Bus 1 and 2 voltages with step change in load on bus 2 in isolated microgrid.

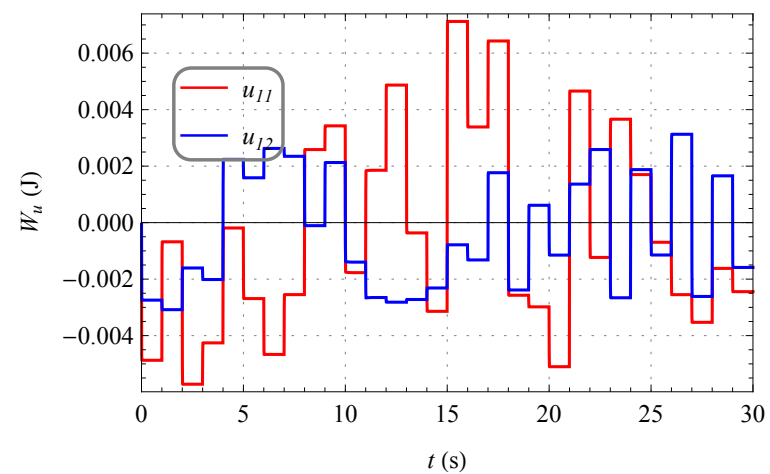

Figure 14: Energy supplied in boost devices storage $u_{1, j}$ in isolated microgrid.

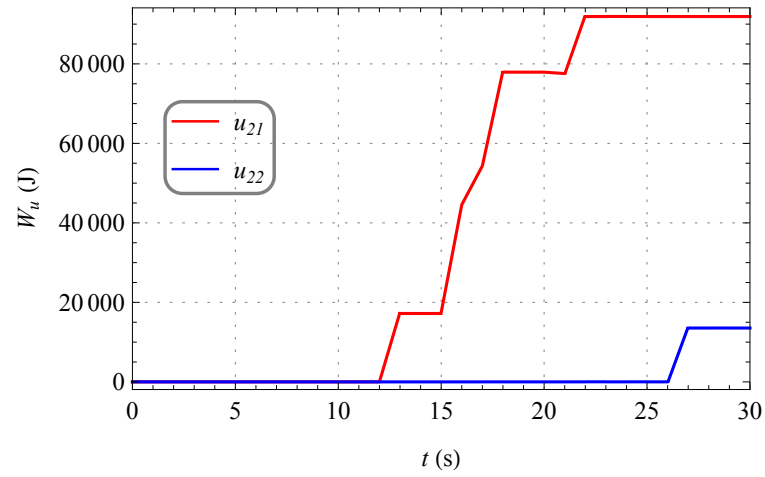

Figure 15: Energy supplied in boost devices storage $u_{2, j}$ in isolated microgrid. 


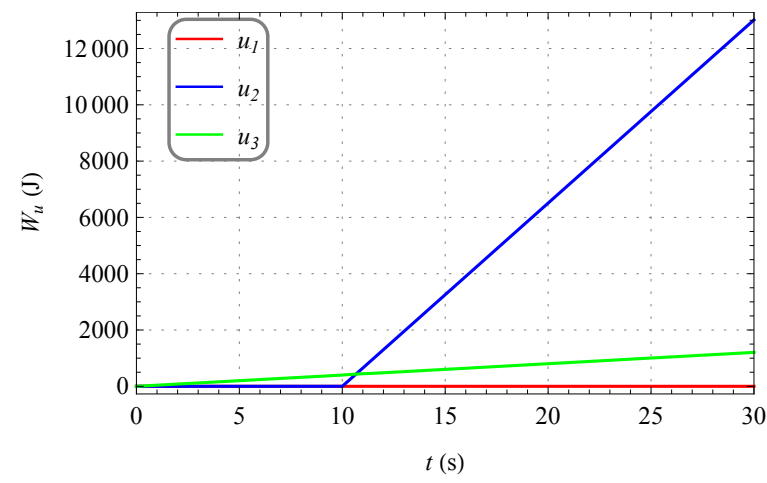

Figure 16: Energy from the bus storage devices in isolated microgrid.

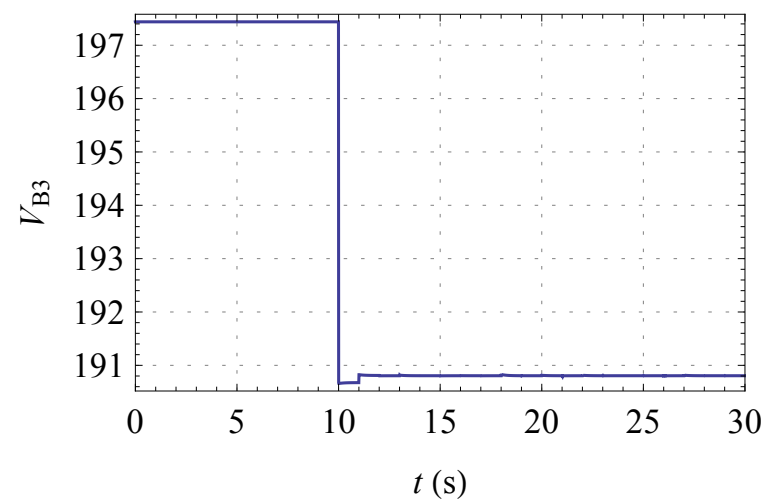

Figure 17: Bus 3 voltage with step change in load on bus 2 in networked microgrid.

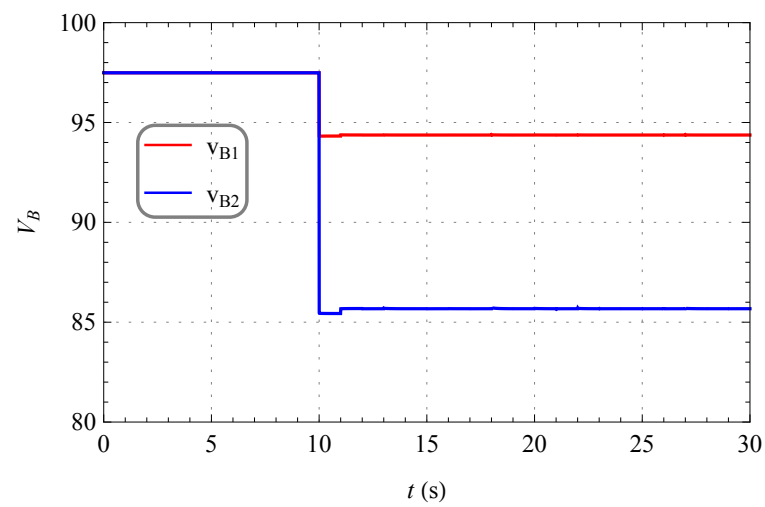

Figure 18: Bus 1 and 2 voltages with step change in load on bus 2 in networked microgrid. 


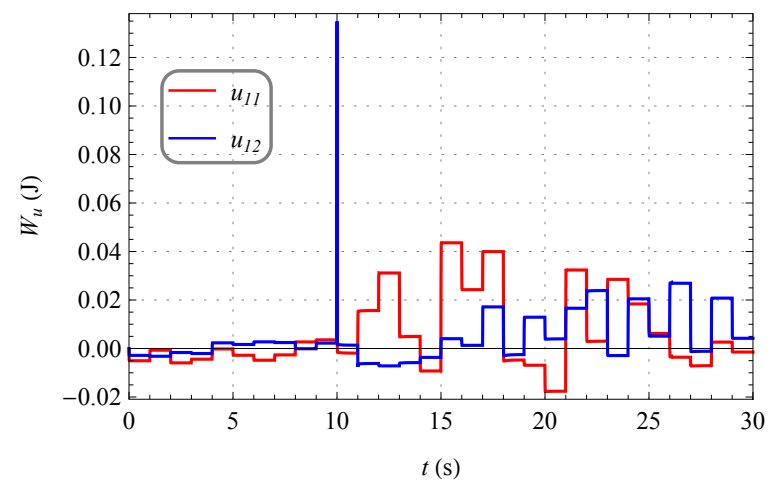

Figure 19: Energy supplied in boost devices storage $u_{1, j}$ in networked microgrid.

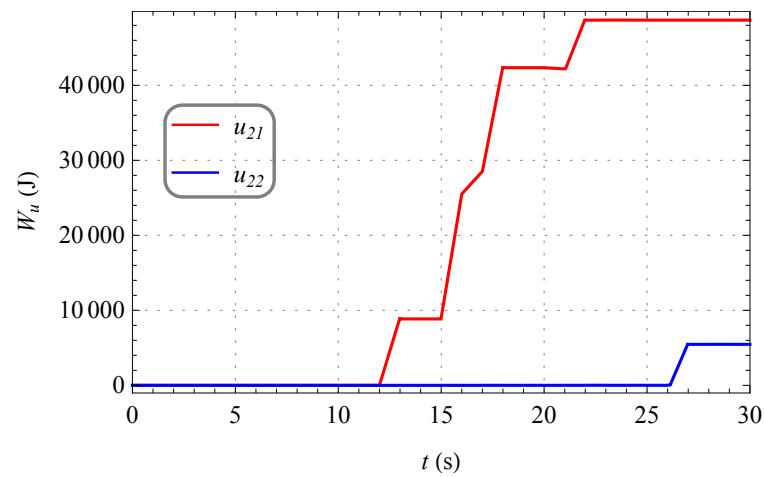

Figure 20: Energy supplied in boost devices storage $u_{2, j}$ in networked microgrid.

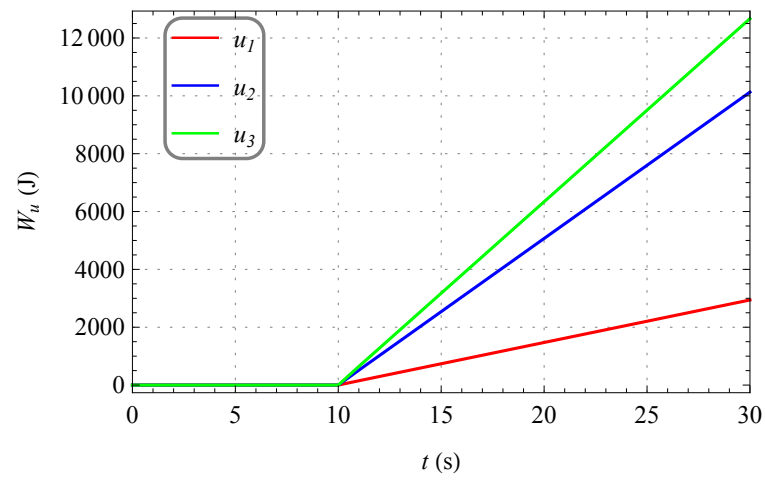

Figure 21: Energy from the bus storage devices in networked microgrid. 


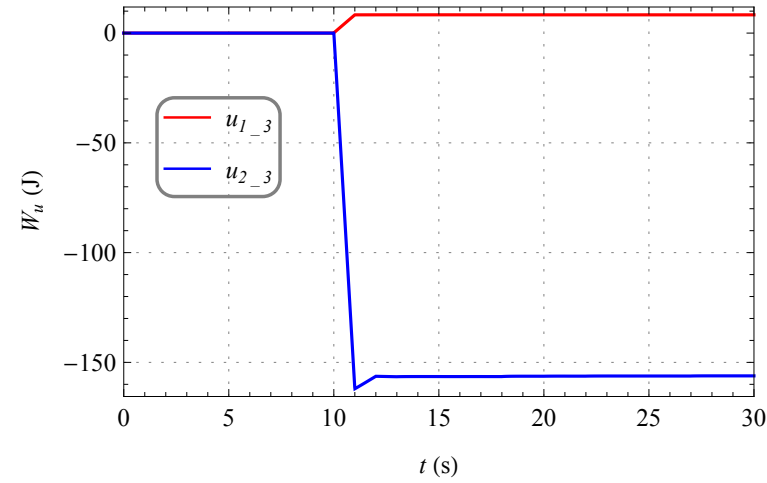

Figure 22: Energy bus interface converters storage $u_{1 \rightarrow 3}$ and $u_{2 \rightarrow 3}$ in networked microgrid.

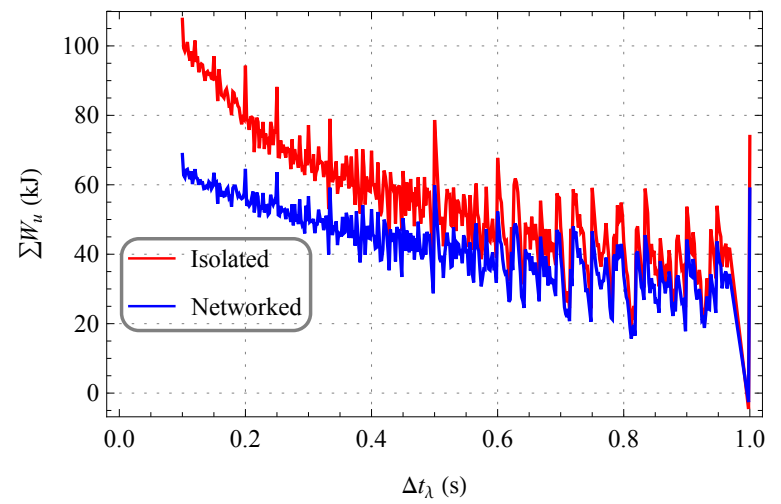

Figure 23: Total energy supplied by enegy storage devices as a function of duty cycle feed-forward update rate. 